\title{
Post-COVID-19 Fatigue: Demographic Distribution and Relation to Chronic Illnesses
}

\author{
Aysha Chaudhary $\mathrm{BS}^{1}$, Jessica Paters $\mathrm{PhD}^{2}$, Mindy Flanagan $\mathrm{PhD}^{2}$, Rachel Pfafman $\mathrm{MPH}^{2}$, \\ Jeanne Carroll RN ${ }^{2}$, Fen-Lei Chang MD PhD ${ }^{1,2}$ \\ Indiana University School of Medicine ${ }^{1}$, Parkview Health Post-Covid Clinic (PPCC) ${ }^{2}$
}

\section{Objective}

This retrospective study examined the relationship between post-COVID (PC) fatigue and the presence of chronic illnesses.

\section{Materials and Methods}

Electronic health records of 122 Parkview PC Clinic patients were reviewed for demographics (age, gender, race), chronic illnesses (diabetes, hypothyroidism, hypertension, congestive heart failure, anemia, cancer,_orthostatic hypotension), obesity (weight, BMI), complaint of fatigue, and activities affected by fatigue. Data was analyzed using Chi-square tests for categorical variables (or Fisher's exact test for small cell sizes) and t-tests for continuous variables. Open thematic coding of activities most affected by PC fatigue was performed.

\section{Results}

95 out of 122 patients $(77.9 \%)$ were found to have PC fatigue. Except for measures related to obesity, none of the chronic illnesses assessed_were correlated with the presence of fatigue. The weight of $P C$ fatigue patients was significantly higher than those without fatigue $(p=0.04)$. Examination of BMI and obesity status also indicated significantly higher BMI $(p=0.01)$ and levels of obesity $(p=0.004)$ in PC fatigue groups compared to the PC non-fatigue groups. No significant differences were observed between the groups in either PT test. Examination of patient described activities effected by PC fatigue found work $(30.4 \%)$, daily stamina $(28.6 \%)$ and ability to exercise (16.7\%) most impacted by PC fatigue. Other activities affected by PC fatigue included housework (5.6\%), hobbies (3.7\%), shopping $(1.9 \%)$ and driving $(1.9 \%)$.

\section{Conclusion}

$77.9 \%$ of our PC patients showed symptoms of fatigue. This is comparable with existing literature. None of the demographic variables and PT tests, or most of the chronic health conditions investigated, were correlated with the presence of fatigue in PC patients. In contrast, measures associated with obesity, including high BMI and weight, were significantly associated with increased PC fatigue presentation. Open thematic coding of qualitative variables indicated work, daily stamina, and exercise were most affected in PC patients. 